\title{
Words with intervening neighbours in infinite Coxeter groups are reduced
}

\author{
HENRIK ERIKSSON* and KIMMO ERIKSSON ${ }^{\dagger}$ \\ Submitted: Aug 5, 2009; Accepted: Jan 20, 2010; Published: Jan 29, 2010 \\ Mathematics Subject Classification: 20F55
}

\begin{abstract}
Consider a graph with vertex set $S$. A word in the alphabet $S$ has the intervening neighbours property if any two occurrences of the same letter are separated by all its graph neighbours. For a Coxeter graph, words represent group elements. Speyer recently proved that words with the intervening neighbours property are reduced if the group is infinite and irreducible. We present a new and shorter proof using the root automaton for recognition of reduced words.
\end{abstract}

\section{Words with intervening neighbours}

Let $G$ be the Coxeter graph of a Coxeter group with generators $S$. Consider a word $w$ in the alphabet $S$.

Definition 1.1. A word has the intervening neighbours property if any two occurrences of the same letter are separated by all its graph neighbours.

In the example below, $s_{0} s_{1} s_{0} s_{2}$ has this property, but $s_{0} s_{1} s_{0} s_{2} s_{1}$ lacks it, since the two occurrences of $s_{1}$ are not separated by the neighbour $s_{3}$.

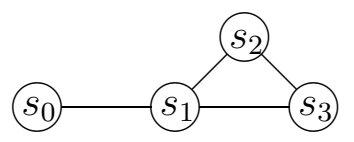

David Speyer [8] recently proved the following result.

Theorem 1.2. For an infinite irreducible Coxeter group, all words with the intervening neighbours property are reduced.

\footnotetext{
${ }^{*}$ School of Computer Science and Communication, Royal Institute of Technology, SE-10044 Stockholm, Sweden. email: henrik@nada.kth.se

${ }^{\dagger}$ School of Education, Culture and Communication, Mälardalen University, Box 883, SE-72123 Västerås, Sweden. email: kimmo.eriksson@mdh.se
} 


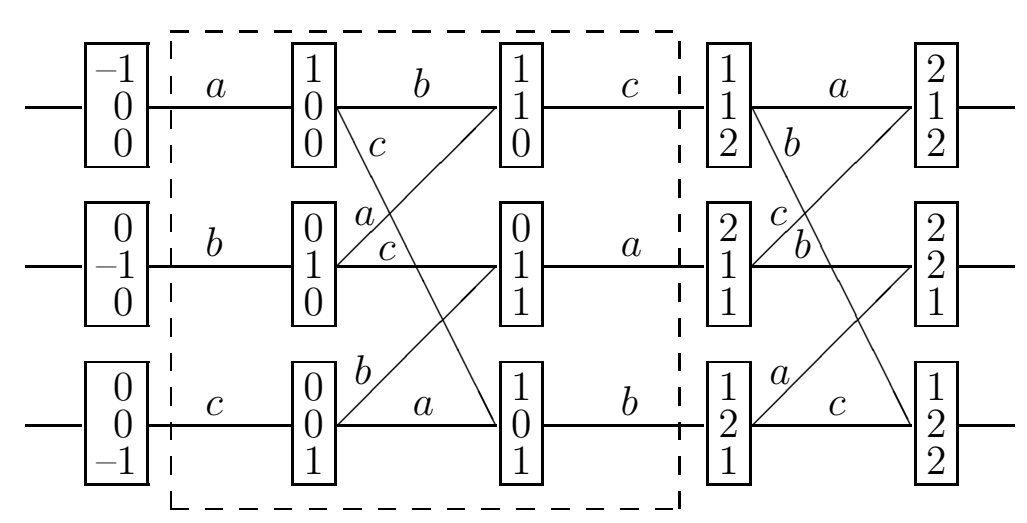

Figure 1: The infinite root poset of $\tilde{A}_{2}$, with the small roots indicated by the dashed box.

In this note, we will demonstrate how the proof of this general result can be reduced to checking the property for just a small family of groups and just a small subset of words, for which verification of the property is straightforward. Our tool will be the finite automaton for recognition of reduced words, invented in [4].

\section{The root automaton}

For any group given by generators and relations, a word $w$ in the generators is called reduced if it is the shortest word for that group element. In general, recognizing reduced words is an undecidable problem. For a Coxeter group, however, a finite recognizing automaton exists [3]. We will here use the concrete root automaton developed by H. Eriksson (for details, see [1]).

In brief, a root in a Coxeter group can be represented as a vector of numbers, one for each vertex of the Coxeter graph. Let $m_{x y} \geqslant 3$ denote the label of the edge between two neighbouring vertices $x$ and $y$ in the Coxeter graph. The set of roots is generated from the unit vectors by sequences of "reflections" indexed by the vertices. The reflection corresponding to a vertex $x$ changes only the $x$-component of the vector; to obtain the new $x$-component, change the sign of its previous value and for each neighbour $y$ of $x$ add the $y$-component value weighted by $2 \cos \left(\pi / m_{x y}\right) \geqslant 1$.

We partially order roots by componentwise $\leqslant$. It is a fundamental fact in Coxeter theory that the nonzero values in a root are either all positive or else all negative, so the poset has a negative side and a positive side.

Figure 2 illustrates the root poset for the affine Coxeter group $\tilde{A}_{2}$, for which the Coxeter graph is a cycle with three vertices, say $a, b, c$, and all edge labels equal to 3 .

In order to interpret the root poset as an automaton, we let words represent paths in the poset: The path starts at the unit root corresponding to the first letter of the word. For each subsequent letter, follow the the corresponding edge in the poset (i.e., perform the corresponding reflection).

All paths start on the positive side (because unit roots are positive). Paths cross over 
to the negative side if and only if the corresponding word may be shortened (reduced) by deletion of its first and the letter where the crossing occurs [1, 4]. For example, in Figure 2 the word $a c a c$ gives a path that ends by crossing to the negative side, so we can delete the first and last letters and obtain $c a$ as a reduced word for the same group element. Thus, we have a deterministic automaton that detects reductions:

Lemma 2.1. A word is reduced if, starting anywhere in the word, the root automaton will never reach a negative root.

The above automaton is of course infinite for infinite groups, but as discussed by Eriksson [4] only a finite set of states is needed. These are the small roots, defined as the roots that can be reached from the unit roots without taking any step that changes a component by 2 or more. For example, the word $a b c$ takes the automaton in Figure 2 through three states:

$$
100 \rightarrow 110 \rightarrow 112 .
$$

The last move increased the $c$-component by 2 , so 112 is not a small root, and in fact there are just six small roots in our example. That an automaton for recognition of reduced words needs the small roots only follows from the following property: When the current state has left the small roots (and reached a "big root"), it will not return to the small roots as long as the word is reduced $[1,4]$. Together with Lemma 2.1, this property implies a result that will serve as our general technique to prove that a word is reduced:

Lemma 2.2. If a word is not reduced then starting somewhere in the word, the root automaton will reach a negative root without previously passing a big root.

\section{Intervening neighbours words in the infinite Cox- eter groups}

As a first illustration of the power of this technique, we will prove Speyer's theorem for the affine group of type $\tilde{A}_{n}$.

Proposition 3.1. For all affine groups of type $\tilde{A}_{n}$, all words with the intervening neighbours property are reduced.

Proof. In order to use Lemma 2.2, take any intervening neighbours word $w$, start anywhere in this word, and follow the corresponding path in the root automaton. To begin with, the 1 in the initial state will propagate to neighbour vertices, then to their neighbours, and so on. Because of the intervening neighbours property, these 1-values will not change before the neighbour 0 -value has been raised. The last 0 -value to be raised has two neighbour 1-values, so the automaton reaches a big root before reaching the negative side.

To cover remaining cases of Speyer's theorem we shall make use of an elementary characterization of infinite Coxeter groups. 
Proposition 3.2. A Coxeter graph defines an infinite group if and only if it has a subgraph isomorphic to one of the graphs in Table 1, possibly with some edge values increased.

Proof. It is straightforward to verify from the classification of finite Coxeter groups (see [1]) that if you start with the graph of a finite group and add an edge (and possibly a vertex), or increase an edge value, you either obtain the graph of another finite group or a graph with a subgraph isomorphic to one of the graphs in Table 1, possibly with some edge values increased.

\begin{tabular}{|c|c|c|c|}
\hline$\tilde{A}_{n}$ & & $\tilde{B}_{n} \quad \mathrm{~A}^{4}-\cdots-\mathrm{O}$ & 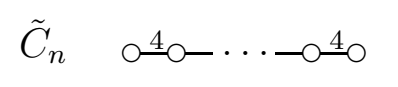 \\
\hline & 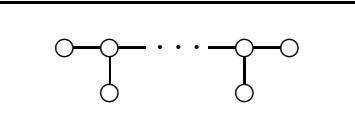 & $\begin{array}{lc}\tilde{E}_{6} & 0-0-9-0-0 \\
0 & 0\end{array}$ & $\tilde{E}_{7} \stackrel{\mathrm{C}}{\mathrm{O}} \mathrm{O}-\mathrm{O}-\mathrm{O}-\mathrm{O}$ \\
\hline$\tilde{E}_{8}$ & 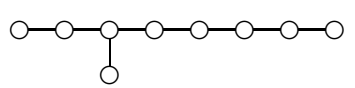 & 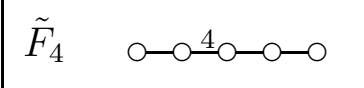 & م-م- \\
\hline
\end{tabular}

Table 1: Coxeter graphs for all affine Coxeter groups and two special hyperbolic Coxeter groups (here nicknamed $\tilde{H}_{3}$ and $\tilde{H}_{4}$ since one obtains them by adding one edge and vertex to the Coxeter graphs of finite groups $H_{3}$ and $H_{4}$ ).

Thanks to the characterization of infinite Coxeter groups in Proposition 3.2, our next two propositions say that it is enough to prove the theorem for the Coxeter graphs in Table 1.

Proposition 3.3. If a Coxeter graph $G$ has the property that all words with intervening neighbours are reduced, this property also holds for the graph $G^{\prime}$ obtained by extending $G$ with a vertex $s^{\prime}$ and an edge $s-s^{\prime}$.

Proof. Consider an intervening neighbours word $w^{\prime}$ in the extended vertex set. We may write $w^{\prime}=w_{0} s^{\prime} w_{1} s^{\prime} w_{2} s^{\prime} \cdots$, where the $w_{i}$ are words in the $G$-vertices. By assumption, the word $w_{0} w_{1} w_{2} \cdots$ is reduced, so it would take the root automaton through a series of positive roots. Now switch to the word $w_{0} s^{\prime} w_{1} w_{2} \cdots$. The difference comes when we play the $s$ that necessarily is in $w_{1}$, for now the extra vertex $s^{\prime}$ may make a positive contribution, say $x$. So $x$ is added to the $s$-component and this effect propagates additively through $w_{1} w_{2} \cdots$. Since this is an intervening neighbours word, all additives will be positive or zero.

The same argument holds for the other occurrences of $s^{\prime}$ in $w^{\prime}$, so the states of the automaton will certainly stay positive. 
Proposition 3.4. If a Coxeter graph $G$ has the property that all words with intervening neighbours are reduced, this property also holds for the graph obtained by increasing an edge label value $s \underline{k}$ t to $s \frac{m}{m} t$, where $k<m$.

Proof. Let $w$ be an intervening neighbours word, so it is reduced over $G$ and takes the automaton through positive roots. Now use the edge label $m$ the first time a value is transported along this edge, from $s$ to $t$, say. The result is a raise of the $t$-value and this effect propagates additively as a positive contribution, when the rest of the word is played. The same argument holds for all later uses of the $m$-label.

So, it remains for us to prove the main theorem for the graphs in Table 1. We have already covered the cyclic case in Proposition 3.1. All the other ten graphs are trees and hence bipartite. For each of these graphs and each start vertex $s$ in that graph, we will define the infinite bicoloured word in the following way. Colour $s$ white, colour all neighbours of $s$ black, colour the neighbours' neighbours white etc, so that black and white vertices alternate. The bicoloured word starts with $s$ followed by all blacks (in any order, they commute!), then all whites, then all blacks etc.

Proposition 3.5. For each of the graphs in Table 1 the bicoloured word has the intervening neighbours property and is reduced.

Proof. The intervening neighbours property of the bicoloured word is immediate from its definition. It is further a very simple verification that for any graph in Table 1 and any start vertex $s$, the bicoloured word takes the root automaton to a big root before it reaches a negative root. (For example, in $\tilde{E}_{6}$ with the center vertex as $s$, we start with a 1 on $s$, then we get 1 on its neighbours, then 2 on $s$, then 2 on its neighbours and finally 4 on $s$, which is a raise by 2 and hence a big root is reached; a Python program for the general case is available at http://www.nada.kth.se/ henrik/intervening.) Therefore the bicoloured word cannot be reducible through deletion of the last letter of any coloured block, but all same-coloured letters commute so in fact the word cannot be reducible at all.

\section{A polygon game that finishes the proof}

The intervening neighbours property is neatly modelled by the chips game. Assuming that the edges of $G$ have been given some orientation, a move consists in choosing a sink node $s$ and reversing all its edge directions, thus making it a source.

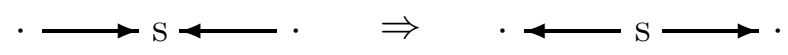

(We call it the chips game because if each arrow-head is detached and pronounced a chip, the rules are equivalent to the chip-firing game of Björner, Lovász and Shor [2].) A fired node cannot be fired again until all neighbours have been fired, so every play sequence will have the intervening neighbours property. In the opposite direction, for any 
intervening neighbours word $w$ and each Coxeter graph edge $s-t$ the neighbours $s$ and $t$ must alternate in $w$ and we may indicate by the arrow $s \rightarrow t$ that it is $t$ 's turn to occur next. Thus, $w$ defines an initial edge orientation such that any edge is directed toward the vertex corresponding to the letter that comes first in $w$. Then, reading each letter of $w$ in turn, it is always the case that the current letter is a sink vertex and after reading it all arrows into it are reversed so that it becomes a source instead [5].

If we interpret the word defined by a chips game as a path in the root poset, we can decide to forbid moves leading to a negative root. This rule defines the positive chips game. A game position then consists of a nonnegative number on each vertex and an orientation of each edge. For every graph $G$ in Table 1 , the infinite bicoloured word is an infinite positive chips play sequence. If we can show that every chips play sequence is, in fact, positive, that is the proof of Theorem 1.2.

If at some stage in a chips game a sink $s$ cannot be played (as that would result in a negative root) then it will remain unplayable, the neighbours of $s$ being deadlocked. But then, after a finite number moves, a terminal position is reached. (The original chipfiring paper [2] expressed this fact as follows: "In an infinite move sequence each node is fired infinitely many times".) But a terminal position cannot occur when there is also an infinite play sequence (the bicoloured one); that statement is true for all polygon games, and we will explain what that means.

It is logically impossible for two neighbour vertices both to be sinks. So, if at any time two different moves are possible, they must involve two non-neighbours and such moves are independent of each other. Thus, the positive chips game is a polygon game (with all polygons being diamonds) in the terminology of K. Eriksson [7]. Here is an example of such a polygon for $\tilde{G}_{2}$ :

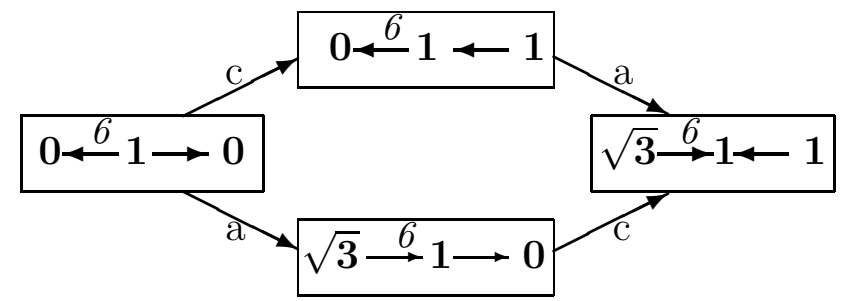

Polygon games have the property that either all play sequences end in the same state after a finite number of moves or all play sequences can be continued indefinitely [7]. Now recall that for each of the ten acyclic graphs in Table 1 the bicoloured word is an infinite path on the positive root poset, corresponding to an initial edge orientation and an infinite play sequence from this initial position. From the polygon property we can conclude that no terminal position occurs and, as we just saw, every chips game with that same initial edge orientation will stay positive, i.e. the corresponding intervening neigbours word is reduced. Our last lemma shows how this result can be extended to all other initial edge orientations.

Lemma 4.1. For each of the ten treelike graphs in Table 1 there is an infinite play sequence in the positive chips game that passes through all possible edge orientations. 
Proof. On a tree, any edge orientation can be transformed into any other edge orientation by some play sequence in the chips game (easily proved by induction, see [5]). Thus, starting from a bicoloured edge orientation we can construct a game that passes through all possible edge orientations. Since the bicoloured play sequence is infinite we know that every game will be infinite, thanks to the polygon property.

Let us now sum up why this means that we have proved what we wanted to prove. Any intervening neighbours word $w$ defines an edge orientation and a play sequence from this edge orientation. From the lemma we know that from this edge orientation an infinite play sequence is possible in the positive chips game. Thanks to the polygon property we then know that all play sequences from this edge orientation can be continued indefinitely and that would not be possible if the $w$-path crossed over to the negative roots.

\section{Discussion and acknowledgments}

We have reproved Speyer's result in an entirely different way, showing the power of the root automaton to deal with questions of reducedness of words in Coxeter groups. We also introduced the roots and chips game that turned out to be a new strongly convergent game; it may be fruitful to investigate other standard questions about such games, cf. [6].

We thank Pavel Tumarkin and Axel Hultman for pointing out to us the need of considering the two special hyperbolic groups in Table 1.

\section{References}

[1] A. Björner and F. Brenti (2005) Combinatorics of Coxeter Groups, Grad. Texts in Math., vol. 231, Springer.

[2] A. Björner, L. Lovász and P. W. Shor, Chip-firing games on graphs, European Journal of Combinatorics 12, 1991, 283-291.

[3] B. Brink and R.B. Howlett (1993) A finiteness property and an automatic structure for Coxeter groups, Mathematische Annalen 296, 179-190.

[4] H. Eriksson (1994) Computational and combinatorical aspects of Coxeter groups, $\mathrm{PhD}$ thesis, KTH.

[5] H. Eriksson and K. Eriksson (2009) Conjugacy of Coxeter elements, Electronic Journal of Combinatorics 16(2), \#R4.

[6] K. Eriksson (1994) Node firing games on graphs, Contemporary Math. 178, 117-127.

[7] K. Eriksson (1996) Strong convergence and a game of numbers, European Journal of Combinatorics 17, 379-390.

[8] D. Speyer (2009) Powers of Coxeter elements in infinite groups are reduced, Proc. Amer. Math. Soc. 137, 1295-1302. 